\title{
Two Confirmed Patients of Anthrax - Binzhou City, Shandong Province, China, August 2021
}

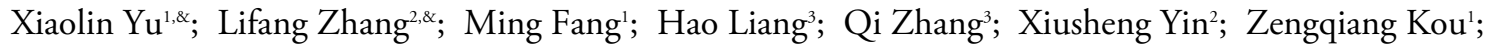 \\ Wenwu Yin ${ }^{4}$; Zhong Li'; Enmin Zhang ${ }^{4, * ;}$; Shujun Ding ${ }^{1, *}$
}

As of August 15, 2021, 2 confirmed cases of anthrax were reported to Shandong CDC, one of which was a 14-year-old student (Patient A) and the other was a 35year-old man (Patient B) engaging in the slaughter of cattle. Patient A died on August 6, and Patient B has been transferred to an infectious disease hospital for isolation and treatment. The Shandong CDC formed a joint investigation team to determine the source of infection and prevent any further spread.

On July 28, 2021, Patient A suddenly experienced fever, fatigue, retching, diarrhea, and convulsions and then was taken to the health clinic of Liumiao Village in the evening of the same day and then again on July 30. On July 31, Patient A was transferred to Binzhou Medical University Hospital due to sudden unconsciousness, lockjaw, and nuchal rigidity during infusion in the village clinic. On August 6, Patient A was voluntarily discharged from the hospital and died the same day. The corpse had been treated under the anthrax disposal regulations, and the burial site had been strictly terminally disinfected. Patient A's cerebrospinal fluid tested positive for Bacillus anthracis, and serum was tested positive for the related $\mathrm{IgG}$. Additionally, the blood culture also tested positive for
Bacillus anthracis by polymerase chain reaction (PCR). Experts preliminarily judged Patient $\mathrm{A}$ as intestinal anthrax or meningitis anthrax caused by sepsis. The genome size of Bacillus anthracis isolated from Patient A was 5,480,063 bp, and guanine-cytosine (GC) content was $35.07 \%$; 5,875 genes were predicted, and the non-coding region accounted for $16.67 \%$ of the total genome (Figure 1). The number of genes annotated to function was 2,679 (45.6\%) (Figure 2). Additionally, this strain included 38 virulence genes (pagA, cya, lef, and atxA, etc.) and complete capsular gene island (capA, capB, cap $C, c a p D$, and capE); 6 drug-resistant genes were found in this genome, including FosB, FosB2, mphL, bla1, bla2, and SatA, all of which were inherent. Mobile genetic elements of this genome included MICBan1, IS231L, ISBce14, IS231T, and ISBce17.

On August 8, Patient B was identified initially as a suspected case during the active investigation and was subsequently diagnosed as cutaneous anthrax by Shandong CDC. Respondents concealed the truth in the early stage of case tracking. On August 12, with the assistance and verification of public security department staff, the investigation team found that

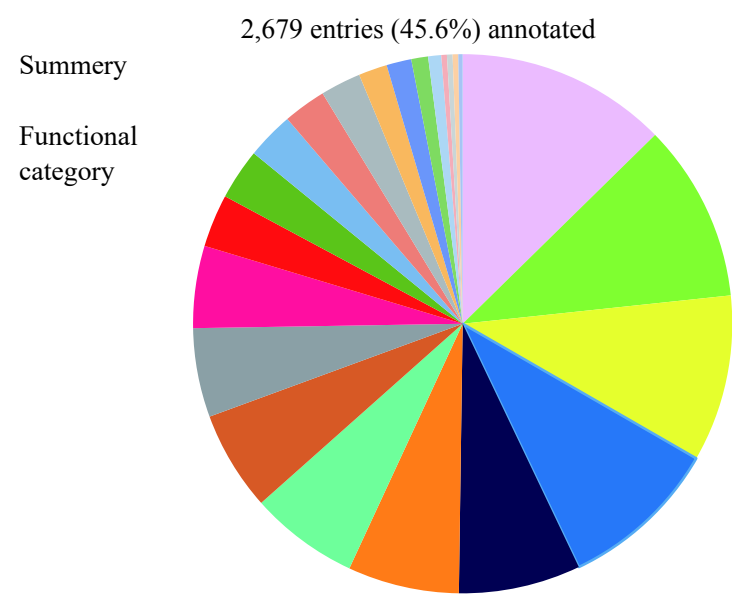

FIGURE 1. Gene function annotation of Bacillus anthracis isolated from Patient A. 


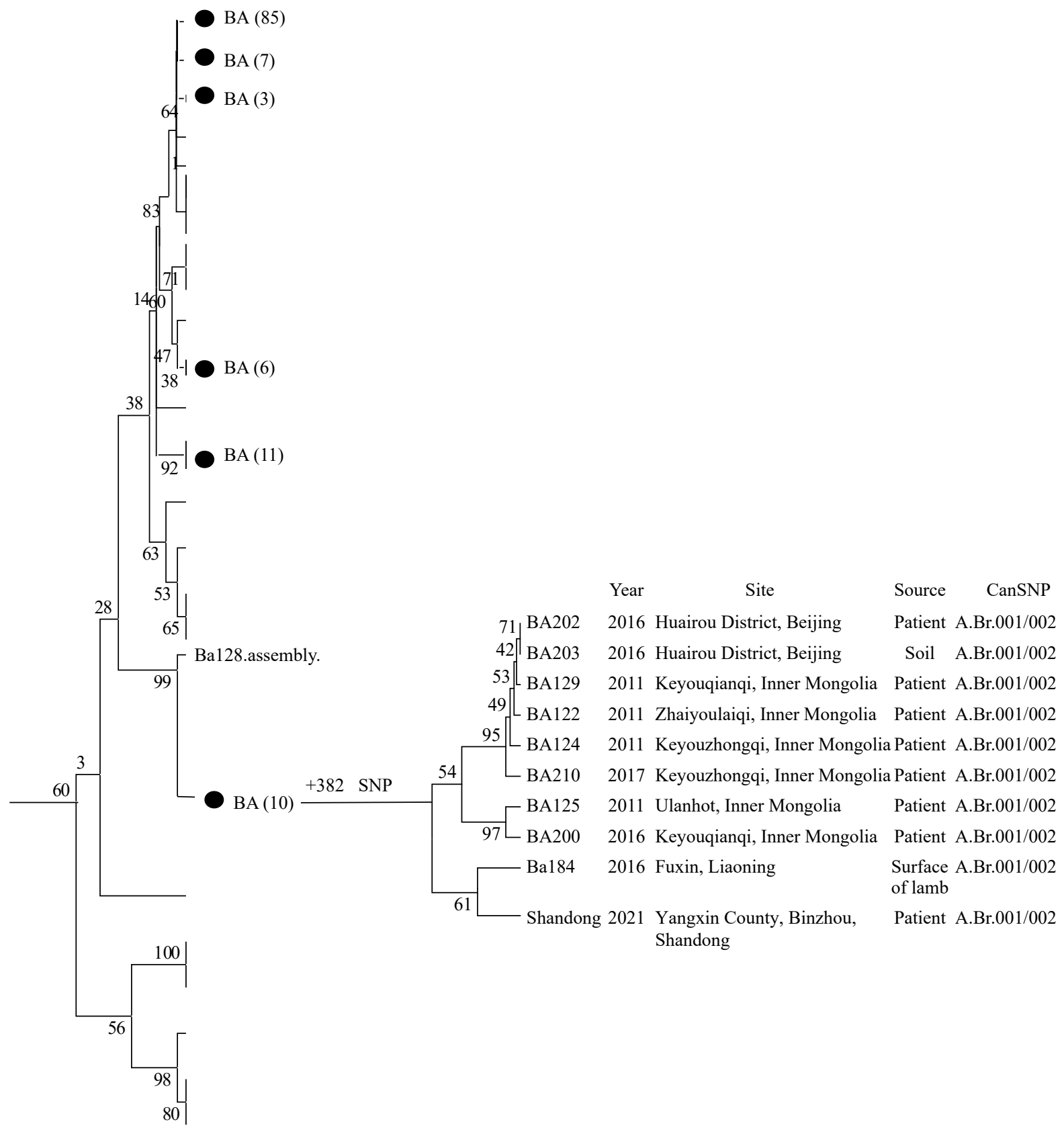

FIGURE 2. Phylogeny of Bacillus anthracis strains based on the unweighted pair-group method with arithmetic mean method.

Patient B slaughtered sick cattle bought from Wendian Town in Yangxin County at Patient A's home on July 25. The cattle seller said that these cattle were purchased from Jilin Province in early July. According to the respondents, black blood flowed on the ground, and internal organs emitted a peculiar smell during the slaughter process. These incidents reflected their lack of awareness of anthrax prevention and control and, when considering their economic burden and the chaotic private trading of livestock (1), laid a considerable risk for anthrax outbreaks.
Shandong CDC and related health agencies implemented a strict 12-day quarantine requirement for all close contacts of the 2 confirmed cases, including 24 that quarantined at home and 9 at centralized isolation facilities. The body temperature and health status of each close contact were monitored daily; meanwhile, 78 people have been screened for fever, diarrhea, vomiting, and skin symptoms in primary medical institutions, and no new cases have yet been found as of August 24. Preventive medications (ofloxacin, ciprofloxacin, etc.) were 
provided to more than 4,700 close contacts and key personnel.

On August 8, a total of 10 serum samples were collected from family members and close contacts of Patient A and other suspected cases, all of which tested negative by enzyme-linked immunosorbent assay test and real-time quantitative PCR. In addition, 13 samples were collected from home environments and smears on the eschar lesions of a suspected cutaneous anthrax case, 8 of which were positive. On August 10, Bacillus anthracis was detected from the ground swab specimens collected from cold storage. For Patient B, investigators collected 160 environment samples (home environment: 21; processing plant: 104; slaughterhouse: 20; feedlot: 15) on August 11, of which 5 samples tested positive for anthrax-specific genes (protective antigen and capsule-related genes). On August 12, nucleic acid testing found that 2 samples collected from the slaughtered sick cattle tested positive.

Investigators checked the cattle ranches and sheep farms simultaneously. As of August 13, 39 blood smear specimens collected from the main testing area involving 8 sheep farms and 39 cattle ranches were all sent to the provincial animal department for testing, and all samples were negative. In addition, 54 households in the key testing area and 69 households in the general testing area were investigated, and investigators found no suspected sick animals. Meanwhile, the county carried out disinfection of farms and slaughterhouses covering 1,254,564 square meters, and the beef products from the families whose samples were previously positive were disposed of properly. On August 14, animal immunization was carried out through Yangxin County.

To find the source of anthrax, prevent the further spread, and eliminate potential risks, the continued investigation and treatment of the current epidemic will be urgent and necessary. To better address the public concern's for anthrax, recommendations have been made to raise awareness for the prevention of anthrax among the public, further strengthening the training and guidance of medical staff and health workers from primary medical and healthcare services.

doi: $10.46234 / \mathrm{ccdcw} 2021.185$

\# Corresponding authors: Enmin Zhang, zhangenmin@icdc.cn; Shujun Ding, dsj_jn@126.com.

\footnotetext{
Shandong Center for Disease Control and Prevention, Jinan, Shandong, China; ${ }^{2}$ Binzhou Center for Disease Control and Prevention, Binzhou, Shandong, China; ${ }^{3}$ Shandong University, Jinan, Shandong, China; ${ }^{4}$ Chinese Center for Disease Control and Prevention, Beijing, China.

\& Joint first authors.
}

Submitted: August 22, 2021; Accepted: August 24, 2021

\section{REFERENCES}

1. Yang XD, Yuan QH, Wu RH. Epidemiology of anthrax in Yunnan, 2000-2015. Dis Surveil 2018;33(7):590 - 3. http://dx.doi.org/10.3784/ j.issn.1003-9961.2018.07.013. (In Chinese). 